\title{
Clinical Audit on Neonatal Care Unit Structure in Five Selected Governmental Hospitals of Addis Ababa, Ethiopia 2019
}

\author{
Asrat Demtse ${ }^{1}$, Girum Sebsibie ${ }^{2 *}$, Yohannes Godie ${ }^{3}$, Yeshi Birhan ${ }^{3}$, Amina Nesru $^{3}$, Askal

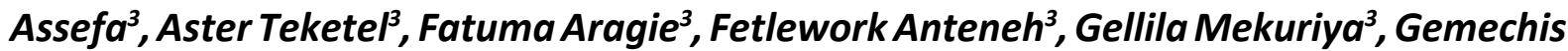 \\ Wari $^{3}$, Hana Endale ${ }^{3}$, Lemlem Berihu ${ }^{3}$, Lensa Habtamu ${ }^{3}$, Mahelet Alemayehu $^{3}$, Misgana \\ Hirpha $^{3}$, Mohammed Tessema ${ }^{3}$, Muluye Dejen ${ }^{3}$, Netsanet ketemaw ${ }^{3}$ and Sitotaw Molla ${ }^{3}$
}

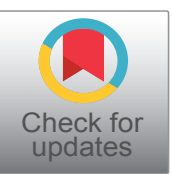

${ }^{1}$ Department of Pediatrics and Child Health, School of Medicine, College of Health Science, Addis Ababa University, Addis Ababa, Ethiopia

${ }^{2}$ School of Nursing and Midwifery, College of Health Science, Addis Ababa University, Addis Ababa, Ethiopia

${ }^{3}$ MSC Students in Neonatal Nursing, School of Nursing and Midwifery, College of Health Science, Addis Ababa University, Addis Ababa, Ethiopia

*Corresponding author: Girum Sebsibie, School of Nursing and Midwifery, College of Health Science, Addis Ababa University, Addis Ababa, Ethiopia

\begin{abstract}
Background: Establishing and/or strengthening Neonatal Intensive Care Unit (NICU) in hospitals includes providing training and continuous support for newborn health care providers and managers. Ensuring the basic equipment's and supplies, strengthening the facility infrastructure and referral system are also mandatory. Clinical audit is the systematic analysis of the quality of health care, including the procedure used for diagnosis, treatment and care, the use of resources and the resulting outcome and quality of life for the patient.
\end{abstract}

Objective: The main objective of this Audit is to evaluate the structure of Neonatal Intensive Care Unit in selected governmental Hospitals in Addis Ababa, Ethiopia.

Methods: The clinical audit was conducted in five purposively selected governmental hospitals in Addis Ababa town. Those hospitals were selected purposively based on the load of their neonatal admission service. Facility based cross-sectional study design was conducted.

Results: Gandhi Memorial Hospital has a neonatal intensive care unit that works independently as one case team under inpatient core processes unit. This hospital works as a delivery unit and maternal health services. About 8,500 delivery service was given in this hospital in 2018 and around 2000 new born admitted in NICU. St. Paul's Hospital and Millennium Medical College: The NICU is equipped with wall thermometer, $\mathrm{O}_{2}$ cylinder and arm pit thermometer. But this equipment's were not found per unit as the room was one and had partition in it. The temperature of the room was kept $22-26{ }^{\circ} \mathrm{C}$ which was similar with the standard. Tikur Anbessa Specialized Hospital has new born corner in the labor \& delivery room and midwife staffs are trained about essential new born care. The NICU is equipped with radiant warmer, incubator, Ambu-bag, suction apparatus, oxygen cylinder; wall thermometer and armpit thermometer. Yekatit 12 hospital had a new born corner in delivery room but there is trained essential new born care staff. The NICU is equipped with radiant warmer incubator, Ambu-bag, suction and oxygen cylinder but wall thermometer, oxygen concentrator, armpit thermometer are not available. Zewditu Memorial Hospital is one of the hospital that give delivery and maternal health service. The delivery unit has its own newborn corner, where the service is given by trained midwives. The NICU is equipped with oxygen cylinder, radiant warmer and armpit thermometer.
Abbreviations
GMH: Gandhi Memorial Hospital; SPHMMC: St. Paul's Hospital and Millennium Medical College; TASH: Tikur Anbessa Specialized Hospital; Y12H: Yekatit 12 Hospital; ZMH: Zewditu Memorial Hospital; CPAP: Continuous Pos- itive Airway Pressure; NICU: Neonatal Intensive Care Unit 


\section{Introduction}

A neonatal intensive care unit (NICU), also known as an intensive care nursery (ICN), is an intensive care unit specializing in the care of ill or premature newborn infants. Neonate refers to the first 28 days of life. Neonatal care, also known as specialized nurseries or intensive care has been started since the 1960s [1].

Newborn intensive care is given for critically ill newborns requiring constant nursing, complicated surgical procedures, regular respiratory support, or other intensive interventions. The first American newborn intensive care unit, designed by Louis Gluck, was opened in October 1960 at Yale New Haven Hospital. NICU is typically directed by one or more neonatologists and staffed by nurses, nurse practitioners, pharmacists, physician assistants, resident physicians, respiratory therapists, and dietitians. Many other ancillary disciplines and specialists are available at larger units [1].

Every day, a significant number of neonates are admitted to neonatal intensive care units (NICU). Some of the main reasons behind NICU referrals are preterm birth and low birth weight. Every year, 15 million infants are born prematurely worldwide [2]. Previous studies determined that the quality of developmental care provided to preterm infants is affected by certain contributing factors, such as nurses' age, years of work experience, educational level and nurse-patient ratio [3].

Clinical audit is the systematic analysis of the quality of health care, including the procedure used for diagnosis, treatment and care, the use of resources and the resulting outcome and quality of life for the patient. Clinical audit is one of the key elements of clinical governance, the latter being a system through which healthcare organizations are accountable for continuously improving the quality of services. It is described as "a framework through which organizations are accountable to continue to improve the quality of the service and safeguard high standards of care by creating environment in which excellence in clinical care would flourish" [4].

In Ethiopia, neonatal health care services have been remaining less consolidated, systematic efforts have been gaining momentum recently. Standard of neonatal health care at all events is now in process and preparedness and readiness of the formal health care system have been in progress. According to the estimates of the American Academy of Pediatrics, nearly $5 \%$ of all the newborns may requires Level III (or Intensive care unit) whilst another $15-20 \%$ of all the newborns may require Level II (Specialty) services [5].

The NICU shall have a clearly identified entrance and reception area for families. On entrance there shall be good acceptance that may make families to feel as part of their babies' health care giver. It should be designed as good security program that protect physical safety of infants, families and staff in the NICU [6]. Hand washing is an important issue in prevention of infection; so there shall be hand washing station in NICU at a distance of not more than $6 \mathrm{~m}$ from each bed of the neonate. Water supply should be available $24 \mathrm{hrs} / 7$ day per week as it is the most important for infection prevention and control in health facilities. Hospital-born babies in developing countries are at increased risk of infections due to poor infection-control practices. Effective infection prevention and control reduces hospital-acquired infections by $30 \%$ [7].

Among physical structure of NICU sound generating activities are the most important that need attention for neonatal wellbeing and safety. But it is difficult to control noise and noise generating activities as NICU noise mostly generated from care activities and professional conduct, such as alarm sounds from different machines. Studies show that incubators can deliver sound that cause deafness of newborn which can be generated by repeated bounces of sound against the hard wall of the dome, amplifying the noise that reaches the neonate $[8,9]$.

An efficient and nurturing physical environment is critical for the support of premature infants, their families, and the healthcare staff that care for them. This clinical audit is to assess the neonatal intensive care unit structure in five purposively selected government hospitals in Addis Ababa. This audit identified potentials problems and challenges encountered on NICU structural service. The audit finding will be important for Baseline information, Policy maker regarding NICU design, staff competence, guidelines development, designing an interventional project towards supplies, configurations, increase existing knowledge and skill of health professional about importance of NICU structure on management of short term and long term problems of new born in the study area. The purpose of the audit is to determine the existing NICU structure in five selected governmental hospitals of Addis Ababa town and to compare the existing structure with predetermined standard.

\section{Methods}

The clinical audit was conducted in five purposely selected government hospitals in Addis Ababa. Those hospitals selected based on their load of neonatal admission. These selected hospitals are Gandhi Memorial hospital (GMH), St. Paul's hospital millennium medical college (SPHMMC), Tikur Anbessa special hospital (TASH), Yekatit 12 hospital medical college (Y12HMC) and Zewditu memorial hospital (ZMH).

GMH is one of those governed by Addis Ababa city administration health bureau and gives maternal and neonatal service. The hospital daily manages $30-40$ delivery cases of pregnant mothers who come from var- 
ious corners of Addis Ababa and nearby towns. In this hospital about eight thousand delivery service were given and about two thousand newborns were admitted in NICU within one year.

SPHMMC is one of the biggest tertiary hospitals in Addis Ababa serving as a referral center for patients from Addis Ababa and most of the western regions of Ethiopia. It provides medical services for more than 250,000 clients every year. It is also a teaching hospital for both undergraduate and postgraduate medical students, being the pioneer in the new integrated teaching curriculum.

TASH is the largest referral hospital in the nation at a tertiary level and its placement covers an area of 4500 meter square. According to human resource statics of hospital, currently it is under Addis Ababa University (AAU) as part of the center of teaching hospital. It has 543 beds and around 2000 patients admitted per month.

Y12HMC: This hospital currently give services like Maternal Health service, Child Health services, Adolescent reproduction Health and the human resource of this hospitals have doctors 19 with specialty, GP 30, BSC nurse 162, diploma nurse 192 and certificate 13, academicians 12 and also other supportive staffs.

ZMH is operated under Addis Ababa health bureau and it is Ethiopia's leading hospital in the treatment of ART patients and currently became the largest HIV clinic in Ethiopia. The hospital has annual delivery of 1800 new born and it has NICU with an average admission of 110 new born per month.

A study was conducted from January to February, 2019 by using observational, structured interview and document review. A hospital based cross-sectional study design was conducted. Data was collected by using observation, interview and document review which are composed of standards on neonatal intensive care unit structures. A data collection was done by the audit teams. The collected data was cleaned and checked for completeness and properly recorded. The final result (report) was presented and discussed in Addis Ababa University, School of Nursing and Midwifery and copy of the audit was sent to each selected hospitals department of NICU.

\section{Results}

This clinical audit is conducted on Neonatal Intensive Care Unit Structure in Five purposively selected governmental hospitals in Addis Ababa. The audit finding from each hospital was presented as follows

Gandhi memorial hospital is one of the hospital that give delivery and maternal health service. The delivery unit has its own newborn corner unit, where the service in newborn corner is given by midwives. Most of midwives in the delivery room had got essential new born training. The newborn corner is equipped with oxygen cylinder and armpit thermometer, but there is no wall thermometer and oxygen concentrator (Table $1 \mathrm{GMH}$ ).

SPHMMC: New born corner is equipped with trained staffs. Essential new born care and advanced life support are well practiced in this ward. This ward was also equipped with wall thermometer, $\mathrm{O}_{2}$ cylinder and armpit thermometer. But this equipment were not found per unit as the room was one and had partition in it. The temperature of the room was kept $22-26{ }^{\circ} \mathrm{C}$ which was similar with the standard (Table $1 \mathrm{SPHMMC}$ ).

TASH: There is new born corner in the labor \& delivery room and all midwives are trained on essential new born care. The room is equipped with radiant warmer incubator, Ambu-bag, suction, oxygen cylinder and wall thermometer and arm pit thermometer (Table $1 \mathrm{TASH}$ ).

Yekatit 12 hospital: New born corner is available in delivery room and it is done by midwives staff. The room is equipped with radiant warmer, incubator, ambu-bag, suction apparatus and oxygen cylinder but wall

Table 1: Delivery and post-natal room staffing in Five Selected Governmental Hospitals at Addis Ababa, Ethiopia, 2019.

\begin{tabular}{|c|c|c|c|c|c|c|c|c|c|c|c|}
\hline \multirow{2}{*}{$\begin{array}{l}\text { Standard and Criteria } \\
\text { Delivery and post-natal room staffing }\end{array}$} & \multirow[t]{2}{*}{ Minimum Criteria } & \multicolumn{2}{|c|}{ GMH } & \multicolumn{2}{|c|}{ SPHMMC } & \multicolumn{2}{|c|}{ TASH } & \multicolumn{2}{|c|}{ Y12 HMC } & \multicolumn{2}{|c|}{ ZMH } \\
\hline & & Yes & No & Yes & No & Yes & No & Yes & No & Yes & No \\
\hline Newborn corner available & $\begin{array}{l}\text { There should be } \\
\text { newborn corner }\end{array}$ & $\sqrt{ }$ & & $\sqrt{ }$ & & $\sqrt{ }$ & & $\sqrt{ }$ & & $\sqrt{ }$ & \\
\hline Staffs trained essential newborn care & $\begin{array}{l}\text { There should be } \\
\text { trained staffs }\end{array}$ & & $\sqrt{ }$ & $\sqrt{ }$ & & $\sqrt{ }$ & & & $\sqrt{ }$ & $\sqrt{ }$ & \\
\hline \multicolumn{12}{|l|}{$\begin{array}{l}\text { Is the room equipped with the following } \\
\text { materials? }\end{array}$} \\
\hline Wall thermometer & One per unit & & $\sqrt{ }$ & & $\sqrt{ }$ & $\sqrt{ }$ & & & $\sqrt{ }$ & & $\sqrt{ }$ \\
\hline $\mathrm{O}_{2}$ cylinder & One per unit & $\sqrt{ }$ & & $\sqrt{ }$ & & $\sqrt{ }$ & & $\sqrt{ }$ & & $\sqrt{ }$ & \\
\hline $\mathrm{O}_{2}$ concentrator & One per unit & & $\sqrt{ }$ & $\sqrt{ }$ & & & $\sqrt{ }$ & $\sqrt{ }$ & & & $\sqrt{ }$ \\
\hline Armpit thermometer & One per unit & $\sqrt{ }$ & & $\sqrt{ }$ & & & $\sqrt{ }$ & & $\sqrt{ }$ & $\sqrt{ }$ & \\
\hline $\begin{array}{l}\text { Is the room temperature kept between } 22- \\
26{ }^{\circ} \mathrm{C} ?\end{array}$ & $22-26^{\circ} \mathrm{C}$ & & $\sqrt{ }$ & & $\sqrt{ }$ & & $\sqrt{ }$ & & $\sqrt{ }$ & & $\sqrt{ }$ \\
\hline
\end{tabular}


thermometer, oxygen concentrator, arm pit thermometer are not available. In addition to this the room is not supplied with electrical power continuously (Table 1 Yekatit 12).

Zewditu memorial hospital is one of the hospital that give delivery and maternal health service. Nearly about 7000 delivery service was given in this hospital in the last 12 months. The delivery unit has its own newborn corner unit, where the service in newborn corner unit is given by midwives. Almost all midwives in the delivery room had trained on essential new born care. The newborn corner is equipped with oxygen cylinder, radiant warmer and armpit thermometer, but there is no wall thermometer so it is difficult to know the room temperature (Table $1 \mathrm{ZMH}$ ).

\section{Newborn care unit layout and physical structure in Five Selected Governmental Hospitals at Addis Ababa, Ethiopia, 2019}

Gandhi memorial hospital: There is a neonatal care unit that stands alone being one case team under inpatient core processes unit. The neonatal care unit is not close to the delivery room but there is an elevator for transportation. The wall and the floor can easily cleanable and the color is white which is appropriate for skin recognition. All the sections of the unit are in one physical area and there are no sound generating activities in or around the unit. But there is poor traffic restriction. The mothers lodge is near to NICU but they haven't access for wheelchair (Table $2 \mathrm{GMH}$ ).

SPHMMC: The neonatal intensive care unit was stand alone as standard and found with the same floor with labor ward at left side. There were adequate bed in NICU relative to the guideline but sometimes more than two babies were kept on one bed which resulted in cross transmission of infection. The wall and the floor can easily cleanable and the color is white which is appropriate for skin recognition. The infants' beds are overcrowded. All the section of the unit is in one corridor and mothers lodge is away from the NICU. There is no

Table 2: Neonatal care unit physical structure in Five Selected Governmental Hospitals at Addis Ababa, Ethiopia, 2019.

\begin{tabular}{|c|c|c|c|c|c|c|c|c|c|c|c|}
\hline Standard and Criteria & Minimum Criteria & GMH & & SPM & AMC & TASI & & Y12 & & ZMH & \\
\hline Neonatal care unit layout and physical & structure & Yes & No & Yes & No & Yes & No & Yes & No & Yes & No \\
\hline Is the NICU stand alone? & Should stand alone & $\sqrt{ }$ & & $\sqrt{ }$ & & $\sqrt{ }$ & & $\sqrt{ }$ & & $\sqrt{ }$ & \\
\hline Is the NICU close to delivery room? & $\begin{array}{l}\text { Should be between labor } \\
\text { ward and postnatal ward } \\
\text { or in post-natal ward or the } \\
\text { same floor with labor ward } \\
\text { or elevator should available } \\
\text { if not on the same floor }\end{array}$ & & $\sqrt{ }$ & $\sqrt{ }$ & & $\sqrt{ }$ & & & $\sqrt{ }$ & $\sqrt{ }$ & \\
\hline Is there adequate bed in the NICU? & 2-3 beds per 1000 deliveries & $\sqrt{ }$ & & $\sqrt{ }$ & & $\sqrt{ }$ & & $\sqrt{ }$ & & & $\sqrt{ }$ \\
\hline $\begin{array}{l}\text { Are the floor and the wall easily } \\
\text { cleanable? }\end{array}$ & Should be washable & $\sqrt{ }$ & & $\sqrt{ }$ & & $\sqrt{ }$ & & & $\sqrt{ }$ & $\sqrt{ }$ & \\
\hline Is the wall color appropriate for NICU? & $\begin{array}{l}\text { White or light for skin } \\
\text { recognition }\end{array}$ & $\sqrt{ }$ & & $\sqrt{ }$ & & $\sqrt{ }$ & & & $\sqrt{ }$ & $\sqrt{ }$ & \\
\hline Does the NICU have window? & One or more & & $\sqrt{ }$ & $\sqrt{ }$ & & $\sqrt{ }$ & & $\sqrt{ }$ & & $\sqrt{ }$ & \\
\hline What is the distance from infant bed? & $0.6 \mathrm{~m}$ from infant bed & & $\sqrt{ }$ & & $\sqrt{ }$ & $\sqrt{ }$ & & $\sqrt{ }$ & & $\sqrt{ }$ & \\
\hline Is air conditioner? & One per unit & & $\sqrt{ }$ & & $\sqrt{ }$ & & $\sqrt{ }$ & & $\sqrt{ }$ & & $\sqrt{ }$ \\
\hline $\begin{array}{l}\text { Do noise generating activities away } \\
\text { from NICU? }\end{array}$ & $\begin{array}{l}\text { Walls, floors, sinks and } \\
\text { ceilings be able to absorb } \\
\text { sound }\end{array}$ & $\sqrt{ }$ & & $\sqrt{ }$ & & $\sqrt{ }$ & & $\sqrt{ }$ & & $\sqrt{ }$ & \\
\hline $\begin{array}{l}\text { Is there appropriate configuration of } \\
\text { the unit? } \\
\text { - All the sections of the unit should } \\
\text { be in one physical area } \\
\text { - Restriction of general traffic } \\
\text { - Availability of dual corridor } \\
\text { - All mothers lodge near NICU } \\
\text { - Babies partitioned into functional } \\
\text { area } \\
\text { - Access for mothers on } \\
\text { wheelchairs } \\
\text { - Access for portable X-ray and } \\
\text { ultrasound machines }\end{array}$ & Not central & $\sqrt{ }$ & $\begin{array}{l}\sqrt{ } \\
\sqrt{ } \\
\sqrt{ }\end{array}$ & $\sqrt{ }$ & $\begin{array}{l}\sqrt{ } \\
\sqrt{ } \\
\sqrt{ } \\
\sqrt{ } \\
\sqrt{ }\end{array}$ & $\begin{array}{l}\sqrt{ } \\
\sqrt{ } \\
\sqrt{ } \\
\sqrt{ } \\
\sqrt{ } \\
\sqrt{ }\end{array}$ & & $\begin{array}{l}\sqrt{ } \\
\sqrt{ } \\
\sqrt{ } \\
\sqrt{ }\end{array}$ & $\begin{array}{l}\sqrt{ } \\
\sqrt{ }\end{array}$ & $\sqrt{ }$ & $\begin{array}{l}\sqrt{ } \\
\sqrt{ } \\
\sqrt{ } \\
\sqrt{ }\end{array}$ \\
\hline
\end{tabular}


wheelchair for mothers, portable X-ray and ultrasound (Table 2 SPHMMC).

Tikur Anbessa specialized hospital: The neonatal intensive care unit is stand alone and has dual corridor. It's close to delivery room. It has adequate bed with each window. Noise generating activities are far from NICU and all section are in one physical area which is restricted from general traffic, all mothers lodge is near NICU and they have access for wheelchair (Table 2 TASH).

Yekatit 12 hospital: The neonatal intensive care unit stands alone which is situated 50 meter away from delivery room and has dual corridor with all section in one physical area which is restricted from general traffic. Noise generating activities are away from NICU and it has adequate bed with the distance of $0.6 \mathrm{~m}$ from each bed and windows. Mothers lodge is near to NICU but they haven't access for wheel chair and wall is not easily cleanable and the color is inappropriate (Table 2 Y12 $\mathrm{HMC}$ ).

Zewditu memorial hospital: There is a neonatal care unit that stands alone as one case team under inpatient directorate. The unit has its own staffs that include one neonatologist, two general practitioners, 22 nurses, two porters and three cleaners. About 55\% of nurses were trained neonatal intensive care. The neonatal care unit is not close to the delivery room but there is an elevator for transportation. The floor and the wall of the unit are easily cleanable, washable and the color of the wall is light to recognize the skin. All the sections of the unit are in one physical area and there are no sound generating activities in or around the unit. But there is poor traffic restriction and the corridor is not dual (Table $2 \mathrm{ZMH}$ ).

\section{Components of neonatal care unit}

Gandhi memorial hospital: The NICU has kangaroo mother care, mothers room and high risk follow up room and isolation room components: -, but there is no milk preparation room in NICU, in KMC room there is no running water for hand washing. Toilets are within easy reach. There are rooms for nurses and doctors but the rooms are not well equipped; doctor's room has no computer and no internet connection, and also nurse's room has no TV and internet connection. The mother restroom and bathroom are not comfortable and attractive (Table $3 \mathrm{GMH}$ ).

SPHMMC: There is no separation room for critical and subcritical room in NICU which is against the standard and all newborn are admitted in the same room except term and preterm separation. There is no isolation room for infectious cases and also no procedure room which is against the standard. This is due to lack of enough room in this unit. There is nurse station and it is not equipped with computer and Internet. There is no room for mothers' and milk preparation in this

Table 3: Components of neonatal care unit in Five Selected Governmental Hospitals at Addis Ababa, Ethiopia, 2019.

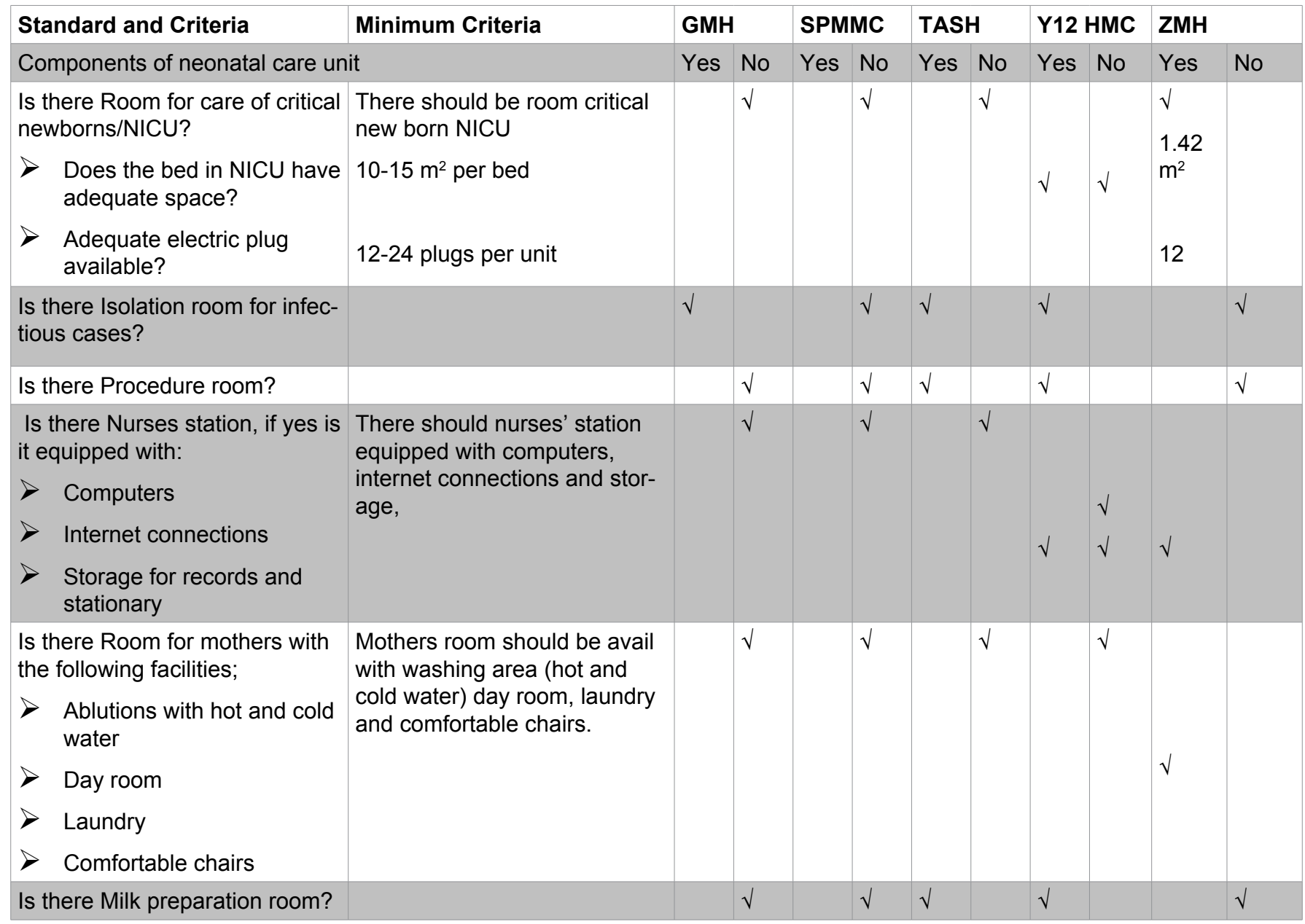


Is there Kangaroo mother care room with adequate space?

Check availability of:

- Feeding cups

- $\quad$ Running water for hand washing

- Toilets are within easy reach

- Bath are within easy reach

- KMC posters

- Follow up in the KMC unit

\section{2-6 beds per a room}

Two for each baby

$>$ One for each room

$>$ One for each room

$>$ One for each room

$>$ Avail in the room

$>$ Four times per day

Is there Toilet and bath for Staff?

Is there Toilet and bath for others?

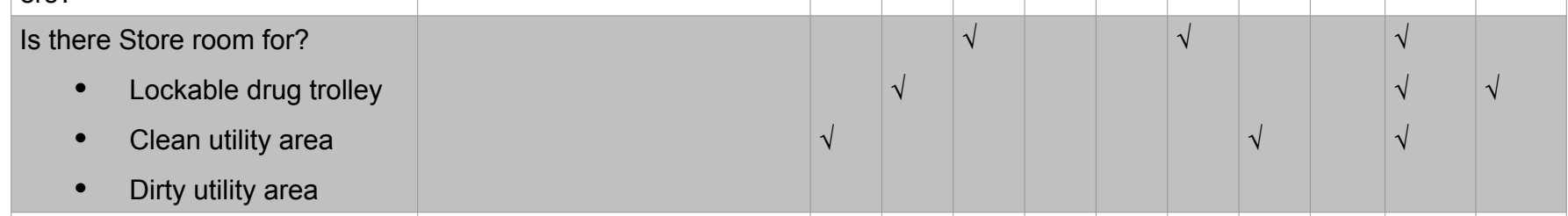

Is there Cleaners area with adequate place to keep cleaning materials?

\begin{tabular}{|c|c|c|c|c|c|c|c|c|c|}
\hline Is there Office for physicians? & $\begin{array}{l}\text { Should equipped with comput- } \\
\text { er and internet connection }\end{array}$ & $\sqrt{ }$ & $\sqrt{ }$ & $\sqrt{ }$ & $\sqrt{ }$ & & $\sqrt{ }$ & $\sqrt{ }$ & \\
\hline $\begin{array}{l}\text { Is there comfortable restroom } \\
\text { for nurses and doctors }\end{array}$ & $\begin{array}{l}\text { The overnight room should } \\
\text { include a bed, table and chair, } \\
\text { internet connection, televi- } \\
\text { sion and en suite bathroom, } \\
\text { restroom with comfortable } \\
\text { chairs, lockers and a dining } \\
\text { area with fridge, microwave } \\
\text { and kettle are required for staff }\end{array}$ & & & $\sqrt{ }$ & $\sqrt{ }$ & & $\sqrt{ }$ & $\sqrt{ }$ & \\
\hline $\begin{array}{l}\text { Is there High risk follow up } \\
\text { room? }\end{array}$ & & $\sqrt{ }$ & & $\sqrt{ }$ & & $\sqrt{ }$ & $\sqrt{ }$ & $\sqrt{ }$ & \\
\hline Is there Sub-critical room? & & $\sqrt{ }$ & & $\sqrt{ }$ & $\sqrt{ }$ & & $\sqrt{ }$ & & $\sqrt{ }$ \\
\hline Is there Phototherapy room? & & $\sqrt{ }$ & & $\sqrt{ }$ & & $\sqrt{ }$ & $\sqrt{ }$ & & $\sqrt{ }$ \\
\hline
\end{tabular}

unit which is against the standard. There is a $\mathrm{KMC}$ room which contains 8 beds that make it over crowded. It has running water, but no bath and toilet which are easy to reach. There is a toilet for staff with bathroom but no bathroom for other clients in the NICU which is against the standard. There is no office for physician due to inadequate room in this unit and also no comfortable restroom for nurses and doctors. There is no high risk follow up in this unit and the follow up carried out at pediatric OPD (Table 3 SPHMMC).

Tikur Anbessa specialized hospital: The NICU has adequate plug and space available, procedure room and physician office with restroom, bathroom, storeroom, high risk follow up, sub-critical room, phototherapy room and KMC room which has feeding cups, KMC posters and follow up in the KMC room. Computer and internet connection are inadequate. There is mother's rest room but not enough. There is no bathroom, isolation room and running water (Table 3 TASH).

Yekatit 12 hospital: The neonatal ICU has isolation room, procedure room, nurse station and physician office with restroom, bathroom, storeroom, high risk follow up, phototherapy room and KMC room which has feeding cups, running water, KMC posters and follow up in the KMC room. Electric plug, computer and internet connection are inadequate. The mother restroom and bathroom are not comfortable and damaged (Table 3 Yekatit).

ZMH: The unit has the following components: Kangaroo mother care; mothers' room and high risk follow up room, but has no isolation room, procedure room and milk preparation room. There are rooms for nurses and doctors but the rooms are not well equipped; doctor's room has computer but has no internet con- 
Table 4: Facilities, supplies and staff competence in Five Selected Governmental Hospitals at Addis Ababa, Ethiopia, 2019.

\section{Standard and Criteria}

Minimum Criteria

\section{Available supply}

Facilities, supplies and staff competence

\section{a. Electricity supply}

\begin{tabular}{|c|c|c|c|c|c|c|c|c|c|c|c|}
\hline $\begin{array}{l}\text { i. Continuous electric supply with } \\
\text { backup }\end{array}$ & $\begin{array}{l}24 \mathrm{hr} / \mathrm{d} \\
7 \mathrm{~d} / \mathrm{wk}\end{array}$ & $\sqrt{ }$ & & & $\sqrt{ }$ & & $\sqrt{ }$ & $\sqrt{ }$ & & $\sqrt{ }$ & \\
\hline $\begin{array}{l}\text { ii. In case of power cut check generator } \\
\text { is automatic or can be started within } \\
5 \text { minute }\end{array}$ & $\begin{array}{l}\text { Should be automatic/ } \\
\text { start within } 5 \text { minutes }\end{array}$ & $\sqrt{ }$ & & $\sqrt{ }$ & & $\sqrt{ }$ & & $\sqrt{ }$ & & $\sqrt{ }$ & \\
\hline iii. Number switch in the unit & One for each light & & $\sqrt{ }$ & & $\sqrt{ }$ & $\sqrt{ }$ & & $\sqrt{ }$ & & $\sqrt{ }$ & \\
\hline $\begin{array}{l}\text { iv. Adequate voltage stabilized out lets } \\
\text { available: Check in the following areas } \\
\text { - In ICU } \\
\text { - } \mathrm{KMC}\end{array}$ & $\begin{array}{l}12 \text { per bed } \\
6 \text { per bed }\end{array}$ & & $\sqrt{ }$ & & $\sqrt{ }$ & & $\sqrt{ }$ & & $\sqrt{ }$ & $\begin{array}{l}12 \\
03\end{array}$ & \\
\hline b. Water supply & & & & & & & & $\sqrt{ }$ & & & \\
\hline $\begin{array}{l}\text { i. Continuous water supply available } \\
\text { with adequate backup source }\end{array}$ & $\begin{array}{l}24 \mathrm{hr} / \mathrm{d} \\
7 \mathrm{~d} / \mathrm{wk}\end{array}$ & & $\sqrt{ }$ & & $\sqrt{ }$ & & $\sqrt{ }$ & $\sqrt{ }$ & & $\sqrt{ }$ & \\
\hline ii. Hand washbasin at entrance & Should available & & $\sqrt{ }$ & $\sqrt{ }$ & & $\sqrt{ }$ & & $\sqrt{ }$ & & & $\sqrt{ }$ \\
\hline $\begin{array}{l}\text { iii. Adequate hand washbasin available } \\
\text { in the unit available }\end{array}$ & One for every $4-6$ babies & & $\sqrt{ }$ & & $\sqrt{ }$ & $\sqrt{ }$ & & $\sqrt{ }$ & & & $\sqrt{ }$ \\
\hline \multicolumn{12}{|l|}{ c. Telephone service } \\
\hline i. Functional telephone in Liaison office & Should be available & $\sqrt{ }$ & & $\sqrt{ }$ & & $\sqrt{ }$ & & $\sqrt{ }$ & & $\sqrt{ }$ & \\
\hline $\begin{array}{l}\text { ii. Telephone service for internal } \\
\text { communication, }\end{array}$ & Should be available & & $\sqrt{ }$ & & $\sqrt{ }$ & & $\sqrt{ }$ & & $\sqrt{ }$ & $\sqrt{ }$ & \\
\hline iii. In the compound for public use & Shall be available & & $\sqrt{ }$ & & $\sqrt{ }$ & & $\sqrt{ }$ & & $\sqrt{ }$ & No & \\
\hline \multicolumn{12}{|l|}{ d. Laboratory service } \\
\hline i. Availability of All the lab tests listed & should be available $24 / 7$ & $\sqrt{ }$ & & & $\sqrt{ }$ & & $\sqrt{ }$ & & $\sqrt{ }$ & No & \\
\hline ii. Turnaround time for $\mathrm{Hgb}$, Blood group & should be $<15$ minutes & & $\sqrt{ }$ & & $\sqrt{ }$ & & $\sqrt{ }$ & $\sqrt{ }$ & & No & \\
\hline
\end{tabular}

Personal protective equipment and IPPS consumables available

\begin{tabular}{|c|c|c|c|c|c|c|c|c|c|c|c|}
\hline iii. Goggle & Five in procedure room be reusable & & $\sqrt{ }$ & & $\sqrt{ }$ & & $\sqrt{ }$ & & $\sqrt{ }$ & No & \\
\hline iv. Doctor's shoes & Two times supply per year for each staff & & $\sqrt{ }$ & $\sqrt{ }$ & & $\sqrt{ }$ & & $\sqrt{ }$ & & $\sqrt{ }$ & \\
\hline V. Apron & Continuous supply & & $\sqrt{ }$ & & $\sqrt{ }$ & & $\sqrt{ }$ & $\sqrt{ }$ & & $\sqrt{ }$ & \\
\hline vi. Gown & Two times supply per year for each staff & $\sqrt{ }$ & & $\sqrt{ }$ & & $\sqrt{ }$ & & $\sqrt{ }$ & & $1 / \mathrm{yr}$ & \\
\hline vii. Surgical face mask & Continuous supply & $\sqrt{ }$ & & $\sqrt{ }$ & & $\sqrt{ }$ & & $\sqrt{ }$ & & $\sqrt{ }$ & \\
\hline viii. Gloves & Continuous supply & $\sqrt{ }$ & & $\sqrt{ }$ & & $\sqrt{ }$ & & $\sqrt{ }$ & & $\sqrt{ }$ & \\
\hline ix. Alcohol & Continuous supply & $\sqrt{ }$ & & $\sqrt{ }$ & & $\sqrt{ }$ & & $\sqrt{ }$ & & $\sqrt{ }$ & \\
\hline X. Chlorine & Continuous supply & $\sqrt{ }$ & & $\sqrt{ }$ & & $\sqrt{ }$ & & $\sqrt{ }$ & & $\sqrt{ }$ & \\
\hline xi. Detergents & Continuous supply & $\sqrt{ }$ & & $\sqrt{ }$ & & $\sqrt{ }$ & & $\sqrt{ }$ & & $\sqrt{ }$ & \\
\hline e. Bed safety & $\begin{array}{l}\text { Should well maintained and covered with } \\
\text { rubber sheet }\end{array}$ & & $\sqrt{ }$ & $\sqrt{ }$ & & $\sqrt{ }$ & & $\sqrt{ }$ & & $\sqrt{ }$ & \\
\hline The sterilization process & $\begin{array}{l}\text { Functional Autoclave/Dry oven should be } \\
\text { available }\end{array}$ & $\sqrt{ }$ & & & $\sqrt{ }$ & & $\sqrt{ }$ & $\sqrt{ }$ & & & $\sqrt{ }$ \\
\hline
\end{tabular}

nection, whereas nurses' room has no TV and internet connection (Table $3 \mathrm{ZMH}$ ).

\section{Facilities, supplies and staff competence}

Gandhi memorial hospital: There is no continuous water supply. But there is continuous supply of electricity with automatic back up. There is no hand washbasin at entrance; instead alcohol hand rub is available. The laboratory service in the unit is poor. There is no list of tests that can be done in the unit. The unit has no its 
own autoclave the sterilization process is done in other ward or in the compound. Regarding personal protective equipment; there is no gown, apron and Doctor's shoes for NICU (Table $4 \mathrm{GMH}$ ).

SPHMMC: There is continuous electric supply with backup start within 5 minutes. Continuous water supply is available without backup and there is sometimes water discontinuation hand wash basin is available at entrance and only one hand wash sink is available. Some of laboratory services are not available in this institution (Table 4 SPHMMC).

TASH: NICU has continuous supply of electricity with automatic back up immediately, numbers of switch are one for each light, liaison office telephone, water supply, personal protective equipment (PPE) like gown, and doctor shoes, except Goggle and apron are available however; laboratory service, laundry service and sterilization services are centrally located for the Hospital. Telephone service is available in the unit for internal communication but currently not functional (Table 4 TASH).

Yekatit 12 Hospital: NICU has continuous supply of electricity with automatic back up, numbers of switch one for each light, water supply, telephone service (liaison office telephone), and personal protective equipment. However, laboratory service, laundry service and sterilization services are centrally located for the hospital. NICU telephone service for internal communication is on process (Table $4 \mathrm{Y} 12 \mathrm{H}$ ).

ZMH: There is continuous supply of water, electricity and consumable equipment. But there is no hand washbasin at entrance and the hand washbasin in the NICU is inadequate; instead alcohol hand rub is available. The laboratory service in the unit is inadequate there is no list of tests that can be done in the unit and all tests done either at central laboratory in the hospital or out of the hospital at private laboratories. The unit hasn't its own autoclave the sterilization process is done in other ward. The supplementation of personal protective equipment is good except gown for NICU, which is supplied only one time per year with its poor quality (Table $4 \mathrm{ZMH})$.

\section{Essential drugs, supplies and equipment should be readily available and accessible}

Gandhi memorial hospital: The number of incubator in this unit is adequate, but phototherapy machine is inadequate. Oxygen blender and Oxygen analyzer equipment is also inadequate. The availability of IV cannula, Syringe \& needle and intravenous fluid is adequate, except $10 \%$ dextrose the mostly wanted IV fluid which not prepared in our country. This $10 \%$ dextrose is prepared manually at the unit. It is time consuming and its prone to contamination so can be one of predisposing factor of neonatal infection. Also in the NICU weight scales, and pulse-oxymeter are available, but CPAP circuit is not functional due to the install problem. In addition to those mentioned above equipment's; Mobile X-ray, portable US, mechanical ventilator, multiparameter monitor, apnea monitor, blood gas analyzer and head boxes are not available in the unit (Table $5 \mathrm{GMH}$ ).

SPHMMC: There are 6 phototherapy machine, 17 functional flow meter, 3 functional incubators and 2 digital weight scale. There is no mechanical CPAP, no Head box, and no mechanical ventilator which is against the standard. Almost all essential drugs and fluid are available except 10\% DW and IV cannula number 24 (Table 5 SPHMMC).

TASH: The neonatal intensive care unit emergency drugs are fully available but there is no chloro-hexidine in this unit and there is no prepared/ready made $10 \%$ dexterous in Ethiopia. The NICU is equipped with medical equipment's like incubator with probes, phototherapy machine, electronic scale, nasal CPAP with circuit, pulse-oximetry, oxygen flow meter and cylinder, IV perfuser, suction machine, resuscitation trolley, neonatal ambu-bags, laryngoscope, endotracheal tube, suction catheter, oxygen tubing, nasal prong, Mobile X-ray, portable US, ventilator, monitor, oxygen and blood gas analyzer and head boxes are available. But Oxygen blenders, Apnea monitor, Multi parameters monitor are not available (Table 5 TASH).

Yekatit 12 hospital: In this hospital the NICU is equipped with medical equipment's like incubator with probes, phototherapy machine, electronic scale, nasal CPAP with circuit, pulse-oximetry, oxygen flow meter and cylinder, perfusers, suction machine, resuscitation trolley, neonatal ambu bags, laryngoscope, ETT, suction catheter, oxygen tubing, nasal prong, neonatal saturation probe. In addition to those mentioned the above equipment's; Mobile X-ray, portable US, ventilator, multi parameter monitor, apnea monitor, oxygen and blood gas analyzer and head boxes are available in the store which is already in the process of installation and also the Hospital is ready to open advanced tertiary level NICU (Table 5 Y12H).

Zewditu memorial hospital: The number of incubator and phototherapy in this unit is inadequate; even though there is no standard for this, more than one newborn are using the same equipment at the same time. Oxygen supplementation equipment and oxygen monitoring equipment are also inadequate. The availability of intravenous fluid is adequate (Table $5 \mathrm{ZMH})$.

\section{Discussion/Comparison with the Standard}

The purpose of this project was to audit the facility readiness and identify potentials problems and challenges of the standard of NICU settings in regards to their equipment, construction costs and space allocation features. Each NICU type may have benefits and drawbacks; and there are various techniques to limit 
Table 5: Essential drugs, supplies and equipment in Five Selected Governmental Hospitals, Addis Ababa, Ethiopia, 2019.

\begin{tabular}{|c|c|c|c|c|c|c|c|c|c|c|c|}
\hline \multirow[t]{3}{*}{ Equipment list } & \multirow{3}{*}{$\begin{array}{l}\text { Minimum } \\
\text { Numbers shall be } \\
\text { Available }\end{array}$} & \multicolumn{10}{|c|}{ Practically Available } \\
\hline & & \multicolumn{2}{|c|}{ GMH } & \multicolumn{2}{|c|}{ SPMMC } & \multicolumn{2}{|c|}{ TASH } & \multicolumn{2}{|c|}{ Y12 HMC } & \multicolumn{2}{|l|}{ ZMH } \\
\hline & & Yes & No & Yes & No & Yes & No & Yes & No & Yes & No \\
\hline Incubator & & 12 & & 03 & & 17 & & & 10 & 02 & \\
\hline Phototherapy machine & & 02 & & 06 & & 17 & & & 6 & 03 & \\
\hline Electronic scale & & 02 & & 02 & & 02 & & $\sqrt{ }$ & 3 & 02 & \\
\hline Ventilators & One per ICU bed & 02 & & 0 & & 03 & & & $\sqrt{ }$ & 0 & \\
\hline Nasal CPAP & One per ICU bed & 02 & & 0 & & 03 & & $\sqrt{ }$ & 4 & 01/bed & \\
\hline Head box & One per bed & 0 & & 0 & & 02 & & $\sqrt{ }$ & 8 & 0 & \\
\hline Pulse-oxymeter & One per bed & 02 & & 03 & & 02 & & $\sqrt{ }$ & 5 & 02/unit & \\
\hline Oxygen flow meter & One per bed & 15 & & 20 & & 18 & & $\sqrt{ }$ & 32 & 01/bed & \\
\hline Oxygen blender & One per bed & 0 & & 0 & & 0 & & & $\sqrt{ }$ & 0 & \\
\hline Oxygen analyzer & One per two beds & 0 & & 0 & & 01 & & & $\sqrt{ }$ & 0 & \\
\hline Apnea monitor & One per two beds & 0 & & 0 & & 0 & & & $\sqrt{ }$ & 0 & \\
\hline IV infusion controllers & One per bed & 06 & & 03 & & 12 & & 9/unit & & 05/unit & \\
\hline $\begin{array}{l}\text { Multi parameters } \\
\text { monitor }\end{array}$ & One per bed & 02 & & 01 & & 0 & & 2/unit & & 01/unit & \\
\hline $\begin{array}{l}\text { Portable suction } \\
\text { apparatus }\end{array}$ & One per six beds & 04 & & 03 & & 06 & & & & 02/unit & \\
\hline Nasal prongs & $\begin{array}{l}\text { Two per oxygen } \\
\text { unit }\end{array}$ & 30 & & 25 & & 50 & & $\sqrt{ }$ & & $02 / \mathrm{O}_{2}$ cylinder & \\
\hline CPAP circuit & $\begin{array}{l}4 \text { circuits per } \\
\text { machine available } \\
\text { for reuse }\end{array}$ & 01 & & 0 & & 02 & & $\sqrt{ }$ & & 0 & \\
\hline $\begin{array}{l}\text { Neonatal saturation } \\
\text { probes }\end{array}$ & $\begin{array}{l}\text { Two per machine } \\
\text { available for reuse }\end{array}$ & 04 & & 0 & & 04 & & $\sqrt{ }$ & & $\begin{array}{l}\text { 01/pulse- } \\
\text { oxymeter }\end{array}$ & \\
\hline $\begin{array}{l}\text { Neonatal incubator } \\
\text { probes }\end{array}$ & Six per incubator & 24 & & 0 & & 30 & & $\sqrt{ }$ & & 02/incubator & \\
\hline IV cannula & Many 24 \& 22G & $\sqrt{ }$ & & 0 & & $\sqrt{ }$ & & $\sqrt{ }$ & & Only $22 \mathrm{G}$ & Not available \\
\hline Feeding cup & Eight per bed & 100 & & 0 & & 100 & & $\sqrt{ }$ & & 03/unit & \\
\hline Chlorhexidine & 05 tubes & 0 & & 0 & & 0 & & & $\sqrt{ }$ & & $\sqrt{ }$ \\
\hline $40 \%$ glucose & 5 ampoules & $\sqrt{ }$ & & $\sqrt{ }$ & & $\sqrt{ }$ & & $\sqrt{ }$ & & 30 ampules & \\
\hline IV Cannula no 24 & 10 in number & $\sqrt{ }$ & & 0 & & 0 & & $\sqrt{ }$ & & $\begin{array}{l}10 \text { each } \\
\text { available }\end{array}$ & \\
\hline Syringe \& needle & $3 \mathrm{cc}, 5 \mathrm{cc}, 10$ each & $\sqrt{ }$ & & $\sqrt{ }$ & & $\sqrt{ }$ & & $\sqrt{ }$ & & $\sqrt{ }$ & \\
\hline IV fluids (crystalloids) & $\begin{array}{l}\text { N/S, } 5 \% \text { DW, } 10 \% \\
\text { DW }\end{array}$ & $\sqrt{ }$ & & $\sqrt{ }$ & & $\sqrt{ }$ & & $\sqrt{ }$ & & 02 tubes & \\
\hline $\begin{array}{l}\text { Tetracycline eye } \\
\text { ointment }\end{array}$ & 5 tubes & 02 & & 02 & & 03 & & $\sqrt{ }$ & & 03 ampoules & \\
\hline Vitamin K & 5 ampoules & 02 & & 02 & & 05 & & $\sqrt{ }$ & & 05 ampoules & \\
\hline Adrenaline & 5 ampoules & 03 & & 05 & & 05 & & $\sqrt{ }$ & & 0 & \\
\hline Nevirapine syrup & Two bottles & 02 & & 0 & & 02 & & $\sqrt{ }$ & & 03 ampule & \\
\hline Aminophylline & 2 ampoules & 06 & & 10 & & 06 & & $\sqrt{ }$ & & 20 tablets & \\
\hline Phenobarbitone & One strip & 10 & & 10 & & 10 & & $\sqrt{ }$ & & 20 tablets & \\
\hline Phenytoin & One strip & 10 & & 10 & & 20 & & $\sqrt{ }$ & & 02 ampoules & \\
\hline Diazepam & Two ampoules & 01 & & 02 & & 03 & & $\sqrt{ }$ & & 0 & \\
\hline
\end{tabular}

the weaknesses in each setting. The review of literature focusing on construction costs reflects the lack of project in this particular area, especially relating to clinical audit structure in NICUs.

Gandhi memorial hospitals: In this Hospital the
NICU is stand alone, and it is not close to delivery room or between the labor and post-natal ward, but the elevator was available. GMH has adequate new born bed and full fills the standard. The floor and the wall are easily cleanable, and the wall color appropriate for NICU. 
The distance of new born bed standard is $0.6 \mathrm{~m}$, but in $\mathrm{GMH}$ less than $50 \mathrm{~cm}$. There is a shortage of equipment like mobile $\mathrm{X}$-ray,, portable suction apparatus, apnea monitor, volumetric pumps and head box. There is also a shortage of oxygen analyzer, laryngoscope, endotracheal tubes, feeding cup and blood gas analyzer. In the structure of NICU in GMH procedure room is not available but isolation rooms for infectious cases are available. Nurse station has no computer, internet connection, storage for records and stationary.

SPHMMC: The neonatal Intensive care unit stands alone as standard and found with the same floor with labor ward at left side. There were adequate bed in this NICU but sometimes more than two babies were kept on one bed which resulted in cross transmission of infection. The wall and the floor can easily cleanable and the color is white which is appropriate for skin recognition and also has window which met the standard. The infants' beds were so closed because of overcrowded which was against the standard.

There is no separation room for critical and subcritical room in NICU which is against the standard and all admitted in the same room except term and preterm separation. There is no isolation room for infectious cases and also no procedure room which is against the standard. This is due to lack of enough room in this unit. There is nurse station and it is not equipped with computer and Internet. in this unit, there is no room for mothers' and milk preparation. There is a KMC room which contain 8 beds that make it over crowded and against the standard. It has running water, but no bath nearby. There is a toilet for staff without bathroom but no bathroom for other clients. There is no office for physician, no comfortable rest room for nurses and Doctors which is equipped with computer and Internet. There is no high risk follow up OPD in this unit and the follow up carried out at pediatric OPD due to overcrowding in this unit which is also against the standard. There is no continuous water supply. Some of laboratory services are not available in this unit.

TASH: There is new born corner in delivery room and there are trained staffs on essential new born care. The neonatal intensive care unit is stand alone and has dual corridor. It's close to delivery room. It has adequate bed with each windows. Noise generating activities are far from NICU and all section are in one physical area which is restricted from general traffic, all mothers lodge are near to the NICU and they have access for wheelchair.

The NICU has adulate plug and space available, procedure room and physician office with restroom, bathroom, storeroom, high risk follow up room, sub-critical room, phototherapy room and KMC room which has feeding cups, KMC posters and follow up in the KMC room. Computer and internet connection are inadequate. They have mother's restroom but not enough.
There is no bathroom, isolation room, nurse station, and running water. The facility have continuous supply of electricity with automatic back up, numbers of switch one for each light, water supply, liaison office telephone, personal protective equipment (PPE). However, laboratory service, laundry service and sterilization services are centrally located for the Hospital.

Yekatit 12 hospital: In this hospital the NICU is staffed with adequate, skilled and trained manpower but there is limitation of equipment like Wall thermometer; $\mathrm{O}_{2}$ concentrator; arm pit thermometers are not fulfilled. The unit is equipped with some essential equipment like incubator; radiant warmer and suction machine. The NICU is placed in isolated area; which is far away from delivery and post-natal ward which is against the standards. The wall and floor is not easily cleanable and painted with non-water proof different blue and weight color.

The unit has adequate windows, located away from noise generating activity, restricted from general traffic, supplied with continuous water and electric source, liaison telephone service neonatal bed with adequate space and covered with rubber as per standards. Many of electric plugs are damaged, mothers have not access of wheelchair, toilet and bath rooms are not fully functional both for the staffs and mothers or visitors. Neonatal intensive care units have separate and isolated rooms for each service as well as staff stations; however, the room doesn't fulfill all requirements as per the set standards; like shortage of ablutions with hot and cold water, toilet, bathroom, comfortable chair, electrical plugs, and computer. There is telephone service for internal communication.

All essential drugs except chlorhexidine, consumable supplies and personal protective equipment are fully available. Also NICU is equipped with medical equipment's there is shortage like incubator with probes, phototherapy machine, electronic scale, nasal CPAP with circuit, pulse-oximetry, oxygen flow meter and cylinder, perfuzers, suction machine, resuscitation trolley, neonatal ambu-bags, laryngoscope, ETT, suction catheter, oxygen tubing and nasal prong. Mobile X-ray, portable US, ventilator, multi parameter monitor, apnea monitor, oxygen and blood gas analyzer and head boxes are available in the store which is already in the process of installation and Yekatit 12 Hospital is ready to open advanced tertiary level NICU.

Zeditu memorial hospital: In the last twelve months nearly about seven thousand delivery services was given in Zewditu memorial hospital (ZMH). A total of 1747 newborn babies were admitted in $\mathrm{ZMH}$ neonatal care unit, out of this total admission $32.7 \%$ were low birth weight (having birth weight less than $2500 \mathrm{~g}$ ). In this hospital neonatal care unit is stand-alone being one case team; the lowest administrative level according to 
this hospital. The rooms were not built for neonatal care purpose initially, so the already available rooms were converted to neonatal care unit with some modifications. Because of this the partitions, corridor, water and sewerage system are not fit to the standard.

The unit is far from delivery room and maternity ward, but there is an elevator near the unit. The numbers of beds in the unit are 12 including two incubators available in the unit. Based on the standard 2-3 beds for 1000 delivery is needed, the total delivery in the institution for the last twelve months was nearly seven thousand. The total delivery of the institution by itself needs $14-21$ which is more than the available beds. So beds in NICU are not adequate when compared to the standard.

The wall and the floor of the unit are easily washable and cleanable, and the adequate wall color is not difficult to recognize the skin color. The NICU has four windows having adequate space from newborn bed $(0.6 \mathrm{~m})$ as stated on the standard. There are only limited machines that can generate noise in the unit and the alarm of the incubators was not noisy; even though, we didn't measure the sound level in the unit. The sound generated outside the room are absorbed by the wall of the block as it is made from concrete which fit with the standard.

The restriction of traffic is poor, there is no adequate lodge for mothers near the unit and there is no wheelchair for mothers. In addition there are no portable X-ray and portable ultrasound. So the configuration of this unit is poor when seen in mirror of the standard. There is room for critical newborns (neonatal intensive care unit) with adequate electric plug in the unit as per the standard. The space of each bed in NICU is about $1.42 \mathrm{~m}^{2}$ which is too much narrow when compared to the standard $\left(10-15 \mathrm{~m}^{2}\right)$.

The unit has no milk preparation room, subcritical room, and phototherapy room, isolation room for infectious disease and procedure room. The room for mothers is also has limited beds, has washing area with only cold water. There is no hot water and laundry. But the standard recommends being available in the unit.

Toilet and bath are available for staff. The nurse station is available in the unit. It is equipped with computer; there is internet connection problem and storage of records and stationary. The KMC room has two beds it is equipped with all materials in the standard. These are in parallel with the standard.

The unit has its own store and areas for clean and dirty utility. But the unit has no lockable drug trolley as well as no area for cleaners and for cleaning materials. There were rooms for nurses and doctors but the rooms haven't comfortable beds, have no TV, have no internet connection, no suite bathroom and other equipment required for staff. These indicate gap when compared to the standard.

Electric supply is good in this unit which is continuously available 24 hours per day from week to week. There is also automatic backup power that starts within five minutes when there is power interruption. There are 12 stabilized voltage outlet in NICU which minimum criteria in the NICU as per the standard. These all are similar with the standard. But in KMC only 03 stabilized voltage outlet are available which fewer in half when compared to the standard.

Water supply is also continuously available 24 hour per day and seven day per week. But there is no hand washbasin at entrance and inadequate hand washbasin the unit when seen in the mirror of the standard. There is functional telephone both liaison and for internal service. So the telephone service is adequate. The laboratory service is poor when compared to the standard. There is no laboratory service in the unit instead; one laboratory is giving service for all hospital departments. The central laboratory is far from the unit and the lists of tests are not available in the unit. The turnaround time for hemoglobin and blood group is greater than $15 \mathrm{~min}$ utes which is too long when compared to the standard.

Almost all personal protective equipment listed in the standard are available with continuous supply. But gown that worn in NICU is supplied only once per year with poor quality which should have to be twice a year according to the standard. This is poor because of its importance for prevention of health care associated infections. The neonatal beds in the unit are safe. Their mattress is covered with rubber sheet that can be easily cleanable. So this met the criteria set in the standard. The sterilization process is not carried out at the unit. There is no dry autoclave/oven in the unit. Because of rare invasive procedure in the unit, they use autoclave available at central point for all the hospital units. When compared to the standard it is inadequate.

In this unit advanced machines used for advanced critical care are not available. For example ventilator machine, head box, oxygen analyzer, oxygen blender, apnea monitor, advanced resuscitation trolley and CPAP circuit are not available. This indicates that the preparedness for advanced critical care is poor when compared to the standard.

In the unit there are two neonatal incubators; when compared to the standard it is not adequate. For this matter there is a time when they admit two newborns in the same incubator at the same time. Three phototherapy machines are available in the unit. This is adequate according to their experience. There are no modern CPAP machines in the unit instead of it they are using locally prepared CPAP which can be avail at any time as per their need. This can be easily prepared at low cost. When compared with the standard it is not satisfactory. 
Equipment such as pulse-oxymeter, oxygen flow meter, intravenous fluid infusion controls, multi-parameters monitor, portable suction apparatus, neonatal saturation probes and neonatal incubator probes are available. But when seen in mirror of the standard all are inadequate in number.

Consumable supplies such as IV cannula (22G \& $24 G), 40 \%$ dextrose, syringe with needles of different size, IV fluids, vitamin k, adrenaline, phenol-barbitone, phenytoin and diazepam are adequate. But lack of $10 \%$ DW and lack of other IV fluids in different volume (125 $\mathrm{ml}, 250 \mathrm{ml}$ and $500 \mathrm{ml}$ ) is the great problem in the unit. This problem is not limited only to this unit it is national problem. Because of $10 \% \mathrm{DW}$ is mostly wanted IV fluid, preparing it in the unit is mandatory. But it is time taking and the procedure makes the fluid prone to microbes. So, fluid supply is not with good quality when compared to the standard.

\section{Conclusion}

All the audited hospitals did not fulfill the pre sated standards of NICU. The physical structure of each hospital and here under the summary of each hospital is stated.

Gandhi memorial hospital: This audit report concluded that among delivery and post-natal room, neonatal care unit lay out and physical structure, component of neonatal care unit, facilities supply and staff competence, essential drug and equipment, staff requirement, essential newborn care guideline and health service information are factors which is audited. The clinical Audit feels that the need for guidelines or protocols of practice in the NICU has been clearly demonstrated. These should be developed by the nursing staff working together as a team so that they feel 'ownership' of the protocols and they should be motivated to comply with them and to continually develop them as practice changes and evolves. Basic guidelines that are applicable to all categories of staff e.g. Hand washing, should be developed as soon as possible so that the staff are aware of how to perform these tasks properly.

SPHMMC: This audit identified that:- Delivery and post-natal room staffing met the minimum criteria needed. There were inappropriate patient to bed ratio and nurse to patient ratio. There were no enough room to separate babies according to their case severity, and also for staffs, \& KMC. There is no enough staff in NICU.

TASH: This audit shows improvement in the availability of basic resources and routine clinical practices. However, errors in prescribing and provision of supportive care and poor data remain a challenge undermining practice and health service monitoring.

Yekatit 12: This audit report concluded that among delivery and post-natal room, neonatal care unit lay out and physical structure, component of neonatal care unit, facilities supply and staff competence, essential drug and equipment, staff requirement, essential newborn care guide line and health service information are part of the audit. There is no trained staff in delivery and post-natal unit, the NICU is not close to delivery room, floor and wall is not easy cleanable and its inappropriate in color and the room has no air conditioner. Scarcity of wall thermometer, $\mathrm{O}_{2}$ concentrator, arm pit thermometer, restroom, bathroom, electric plug, Telephone service, laboratory service sterilization process and laundry are problem of the unit.

ZMH: The delivery and post-natal room staffing is good. But the criteria needed in delivery room and newborn corner unit is not in line with the standard. Among components of neonatal care unit $40 \%$ of them are not available at all, whereas about $60 \%$ of components are available but these are incomplete. Facilities and supplies are good and equipment that are used for essential new born care are also available. There was no hand washbasin at entrance and the hand washbasin in NICU is in adequate. Equipment used for advanced critical care is almost not available. Nurse to patient ratio is not according to the standard in this unit.

\section{References}

1. Oestergaard MZ, Inoue M, Yoshida S, Mahanani WR, Gore FM, et al. (2011) Neonatal mortality levels for 193 countries in 2009 with trends since 1990: A systematic analysis of progress, projections, and priorities. PLoS Med 8: e1001080.

2. Kenya National Bureau of Statistics (KNBS), ICF Macro (2010) Infant and child mortality. In: Kenya demographic and health survey 2008-09. Calverton, Maryland, USA.

3. Ministry of Health, Government of Kenya (2017) The Kenya Health Sector Strategic and Investment Plan-KHSSP from July 2012-June 2017.

4. WHO (2009) Pregnancy, childbirth, postpartum and newborn care: A guide for essential practice.

5. EMOH, Ethiopian Neonatal Intensive Care Unit (NICU) (2014) Training participants' manual.

6. American Academy of Pediatrics, Joint Committee on Infant Hearing (2007) Year 2007 position statement: Principles and guidelines for early hearing detection and intervention programs. Pediatrics 120: 898-921.

7. Central Statistical Agency [Ethiopia], ICF International (2012) Ethiopia demographic and health survey 2011. Addis Ababa, Ethiopia; Calverton, MD, USA.

8. Megan Doede, Alison M Trinkoff, Ayse P Gurses (2018) Neonatal intensive care unit layout and nurses' work. HERD 11: 101-118.

9. Chomba E, McClure EM, Wright LL, Carlo WA, Chakraborty $\mathrm{H}$, et al. (2008) Effect of WHO newborn care training on neonatal mortality by education. Ambul Pediatr 8: 300-304.

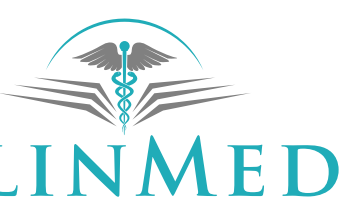

INTERNATIONAL LIBRARY 